\title{
Lucille Beaudry, Marc Chevrier (sous la direction de), Une pensée libérale, critique ou conservatrice?
}

\section{Simona Rossi}

\section{(2) OpenEdition}

12 Journals

\section{Edizione digitale}

URL: http://journals.openedition.org/studifrancesi/9698

DOI: 10.4000/studifrancesi.9698

ISSN: 2421-5856

\section{Editore}

Rosenberg \& Sellier

\section{Edizione cartacea}

Data di pubblicazione: 1 décembre 2007

Paginazione: 703-704

ISSN: 0039-2944

\section{Notizia bibliografica digitale}

Simona Rossi, «Lucille Beaudry, Marc Chevrier (sous la direction de), Une pensée libérale, critique ou conservatrice?», Studi Francesi [Online], 153 (LI | III) | 2007, online dal 30 novembre 2015, consultato il 12 janvier 2021. URL: http://journals.openedition.org/studifrancesi/9698 ; DOI: https://doi.org/ 10.4000/studifrancesi.9698

Questo documento è stato generato automaticamente il 12 janvier 2021.

\section{(c)}

Studi Francesi è distribuita con Licenza Creative Commons Attribuzione - Non commerciale - Non opere derivate 4.0 Internazionale. 


\title{
Lucille Beaudry, Marc Chevrier (sous la direction de), Une pensée libérale, critique ou conservatrice?
}

\author{
Simona Rossi
}

\section{NOTIZIA}

LUCILLE BEAUDRY, MARC CHEVRIER (sous la direction de), Une pensée libérale, critique ou conservatrice?, Montréal, Les Presses de l'Université Laval, 2007, pp. 220.

1 Il Québec nasconde un'anima liberale o conservatrice? È a questo ampio quesito che intendono dedicarsi gli autori di questo testo, i quali esplorano con zelo e audacia i repentini cambiamenti che sono intervenuti sulla scena politica quebecchese dalla Rivoluzione Tranquilla ad oggi. Nella convinzione che il mondo della politica conosca un'evoluzione per nulla casuale, influenzata da regole ben precise e da "ondate" di pensiero ora in salita, ora in discesa, essi scelgono d'illustrare il percorso politico quebecchese del xx secolo attraverso il filtro di tre grandi pensatori del Novecento: Emmanuel Mounier, Hannah Arendt e George Grant. Teorici politici di spicco e caparbi oratori, in grado di fare presa sulle folle, questi tre intellettuali hanno dato un importante contributo alla formazione del variegato pensiero politico quebecchese.

2 Il primo rappresenta un vero e proprio punto di riferimento: Lucille BEAUDRY, FélixOlivier RIENDEAU e Martin MEUNIER spiegano come egli abbia sempre sostenuto gli anticlericali e come i suoi comizi abbiano dato loro la forza e gli strumenti necessari, negli anni della Rivoluzione Tranquilla, per «renverser un ordre social et lui en substituer un autre» (p. 27). Hannah Arendt, invece, una donna forte e impegnata nel sociale, è stata la musa ispiratrice della nuova generazione sorta dai disordini degli anni Sessanta. Jean-Pierre coUtURE e Francis mOREAUlt descrivono e analizzano il suo originale concetto di "politica globale", che abbatte finalmente la tradizionale frontiera tra politico e apolitico, coinvolgendo ogni sfera del vivere individuale e collettivo. 
George GRANT, infine, è molto apprezzato e conosciuto nel Canada anglofono, mentre, come spiegano Yves COUTURE, Éric BÉDARD e Marc CHEVRIER, è stato recepito piuttosto tardi in Québec, poiché il suo conservatorismo, basato sulla negazione del progresso tecnologico, non ha incontrato per molto tempo il favore della popolazione quebecchese, ansiosa di mettersi alla pari degli Stati Uniti dal punto di vista pratico ed economico.

3 Questi tre diversi "percorsi" di pensiero, con tutte le loro sfaccettature, conducono gli autori di quest'opera ad affermare che il Québec, più che liberale o conservatore, è più che altro un paese idealista e "pluralista", costretto nel passato ad accettare rigide imposizioni e per questo oggi mai pago di cercare diverse soluzioni e teorie di ampio respiro. Un testo ben strutturato e di gradevole lettura, che di certo può aiutare il lettore a meglio districarsi nella lettura della realtà politica quebecchese dei tempi moderni. 